\title{
Moving Towards Therapy in SCA1: Insights from Molecular Mechanisms, Identification of Novel Targets, and Planning for Human Trials
}

\author{
Sharan R. Srinivasan ${ }^{1} \cdot$ Vikram G. Shakkottai $^{1,2}{ }^{(B)}$ \\ Published online: 23 July 2019 \\ (C) The American Society for Experimental NeuroTherapeutics, Inc. 2019
}

\begin{abstract}
The spinocerebellar ataxias (SCAs) are a group of neurodegenerative disorders inherited in an autosomal dominant fashion. The SCAs result in progressive gait imbalance, incoordination of the limbs, speech changes, and oculomotor dysfunction, among other symptoms. Over the past few decades, significant strides have been made in understanding the pathogenic mechanisms underlying these diseases. Although multiple efforts using a combination of genetics and pharmacology with small molecules have been made towards developing new therapeutics, no FDA approved treatment currently exists. In this review, we focus on SCA1, a common SCA subtype, in which some of the greatest advances have been made in understanding disease biology, and consequently potential therapeutic targets. Understanding of the underlying basic biology and targets of therapy in SCA1 is likely to give insight into treatment strategies in other SCAs. The diversity of the biology in the SCAs, and insight from SCA1 suggests, however, that both shared treatment strategies and specific approaches tailored to treat distinct genetic causes of SCA are likely needed for this group of devastating neurological disorders.
\end{abstract}

Key Words SCA1 $\cdot$ Spinocerebellar ataxia $\cdot$ antisense oligonucleotides $\cdot$ potassium channels $\cdot$ biomarkers $\cdot$ clinical trials.

\section{Introduction}

Spinocerebellar ataxias (SCA) are a group of autosomal dominant degenerative disorders causing progressive decline in gait, balance, and speech and are often associated with eye movement abnormalities [1,2]. Several of these conditions are associated with pyramidal or extrapyramidal symptoms, and many are associated with sensory neuropathy.

New genes causing SCA are annually discovered, and the list of SCAs continues to grow. The number of SCAs is now in the high forties. Although the true incidence is difficult to determine given the lack of identified causal genes for several of the SCAs, SCA is estimated to affect

Vikram G. Shakkottai

vikramsh@med.umich.edu

1 Department of Neurology, University of Michigan, Ann Arbor, Michigan, USA

2 Department of Molecular and Integrative Physiology, University of Michigan, 4009 BSRB, 109 Zina Pitcher Place, Ann Arbor, Michigan 48109, USA
1 to 5 in 100,000 people [2]. The most common SCAs result from an expansion of a glutamine encoding CAG repeat in the respective disease genes. Epidemiologic data for the so called polyglutamine (polyQ) SCAs have been determined by the Clinical Research Consortium for SCAs (CRC-SCA) in North America and the European Integrated Project on SCAs (EUROSCA) [3, 4]. The natural history data obtained by these consortia have indicated that SCA type 1 (SCA1) is the most rapidly progressive SCA subtype, with disease onset typically in the third or fourth decade of life $[3,4]$.

Like many SCAs, SCA1 consists of progressive gait ataxia, dysarthria, and later bulbar dysfunction [2]. As SCA1 typically spares the pars compacta of the substantia nigra, other elements of the basal ganglia, and the thalamus, extrapyramidal symptoms are less prominent $[5,6]$. In later stages of the disease, cognitive deficits, specifically memory impairments and executive dysfunction, have been reported [7, 8].

Although clinical history and examination are crucial elements of any neurological disease, definitive diagnosis of SCA depends on genetic testing. In disorders such as SCA1, targeted polymerase chain reaction (PCR) is the most costeffective way to identify the repeat expansion. 


\section{Pathophysiology of SCA1}

SCA1 stems from an expanded CAG repeat in the ATXN1 gene. Expansion beyond $39 \mathrm{CAG}$ repeats is pathologic. Longer repeats generally result in earlier onset of disease. For SCA1, CAT interruptions can alter the penetrance and aggressiveness of disease [9].

It should also be noted that although polyQ repeats are considered causal for pathology, glutamine expansion and dysfunction of the ATXN1 protein itself is not sufficient. Numerous studies have shown that ATXN1 interacts with transcription regulators, RNA splicing factors, and other nuclear receptors to drive cerebellar Purkinje cell dysfunction [10-12]. ATXN1 has a nuclear localization signal (NLS) and in its wild-type state shuttles between nucleus and cytoplasm [13]. Conversely, polyQ expanded ATXN1 is partitioned largely in the nucleus [14]. Noteworthy though is that polyQ ATXN1 without a functioning NLS does not lead to a disease phenotype, suggesting that toxicity is exerted in the nucleus, likely through transcriptional dysregulation. Another feature of ATXN1 is a phosphorylation site on S776, required for stabilization of polyQ ATXN1. In fact, ATXN1(82Q)-S776A will not lead to a disease phenotype, although ATXN1(30Q)S776D does $[15,16]$, suggesting that this ATXN1 stabilization is mandatory for manifesting disease.

The combined interactions between mutant ATXN1 and its binding partners alters expression of surface receptors and ion channels, working to perturb membrane excitability. In mouse models of disease, alterations in cerebellar Purkinje cell spiking due to ion channel dysfunction occurs concurrently with motor impairment, and well before cell loss [17-19].

In later stages of SCA1, histopathology shows degeneration of brainstem and cerebellar neurons, classically thought of as olivopontocerebellar atrophy [5], though recent studies have shown involvement in other regions indicated above [20]. Most of the existing research in SCA1 has been restricted to understanding cerebellar dysfunction and degeneration. Premature mortality in SCA1 is, however, likely related to brainstem dysfunction. Studies have only recently been initiated to understand transcriptional dysregulation in the brainstem in SCA1 [21, 22]. It is likely that the transcriptional targets of polyQ ATXN1 differ in the cerebellum and brainstem.

\section{Treatment}

Developing therapeutics for neurodegenerative diseases has proven a difficult task despite considerable efforts. In designing any therapeutic, it is vital to understand the purpose behind the intervention. What is to be gained from this treatment? How does it address an unmet or poorly met clinical concern? Do the benefits outweigh any potential adverse effects?
As we work towards developing treatments for SCAs, it is important to consider both symptomatic therapy as well as disease modifying therapy (DMTs). The latter, although certainly the ideal goal, may be more difficult. Further, in focusing on helping patients, we should not be limited in our efforts to alleviate symptoms at the cost of pursuing DMTs. Indeed, addressing symptomatic dysfunction may actually serve as means of modifying clinical course. A particularly salient example of this exists already within the movement disorders field. Dopamine replacement therapy has been the mainstay in treatment of patients with idiopathic Parkinson disease (iPD). Levodopa produces profound improvement of patient symptoms (i.e., bradykinesia, tremor, and rigidity). Although currently debated, long-term administration of levodopa may also slow disease progression [23].

\section{Targets for Treatment}

\section{Identifying a Suitable Target for SCA1}

There are several elements to consider in identifying a proper drug target for neurological diseases. Identifying targets specific to not only the central nervous system (CNS) but unique to the substructures (e.g., brainstem or cerebellar nuclei) is pertinent. This is not strictly necessary, though, and several treatments have circumvented target specificity through use of adjusting pharmacokinetics and pharmacodynamics. The best example of this again is carbidopa/levodopa in iPD, which provides a substrate for dopamine signaling but without specificity against CNS dopamine receptors. In fact, without carbidopa to prevent systemic metabolism of levodopa, this drug was considered a failure due to intolerable side effects (largely gastrointestinal) from conversion into dopamine in the periphery. Thus, although target specificity is not a strict requirement, the treatment in question certainly must act on the target within the CNS to be effective. This requires blood-brain barrier permeability (increased lipophilicity) although maintaining aqueous solubility for treatment delivery (increased hydrophilicity). One must therefore consider not simply a suitable target structure, but also the substructure binding site or mechanism of intervention. Packaging and delivery therefore become as important as the actual therapeutic itself [24]. An important consideration with any therapy is the risk for off-target effects. These must be kept in mind as we work towards developing new therapeutics. Recent work in SCA1 has identified several potential targets for therapy (Fig. 1) discussed below. As we evaluate these targets though, we must consider the factors discussed above.

In discussing treatment, it is important to recognize that timing of treatment delivery is very important. Earlier treatment has proven efficacy in delaying progression and 


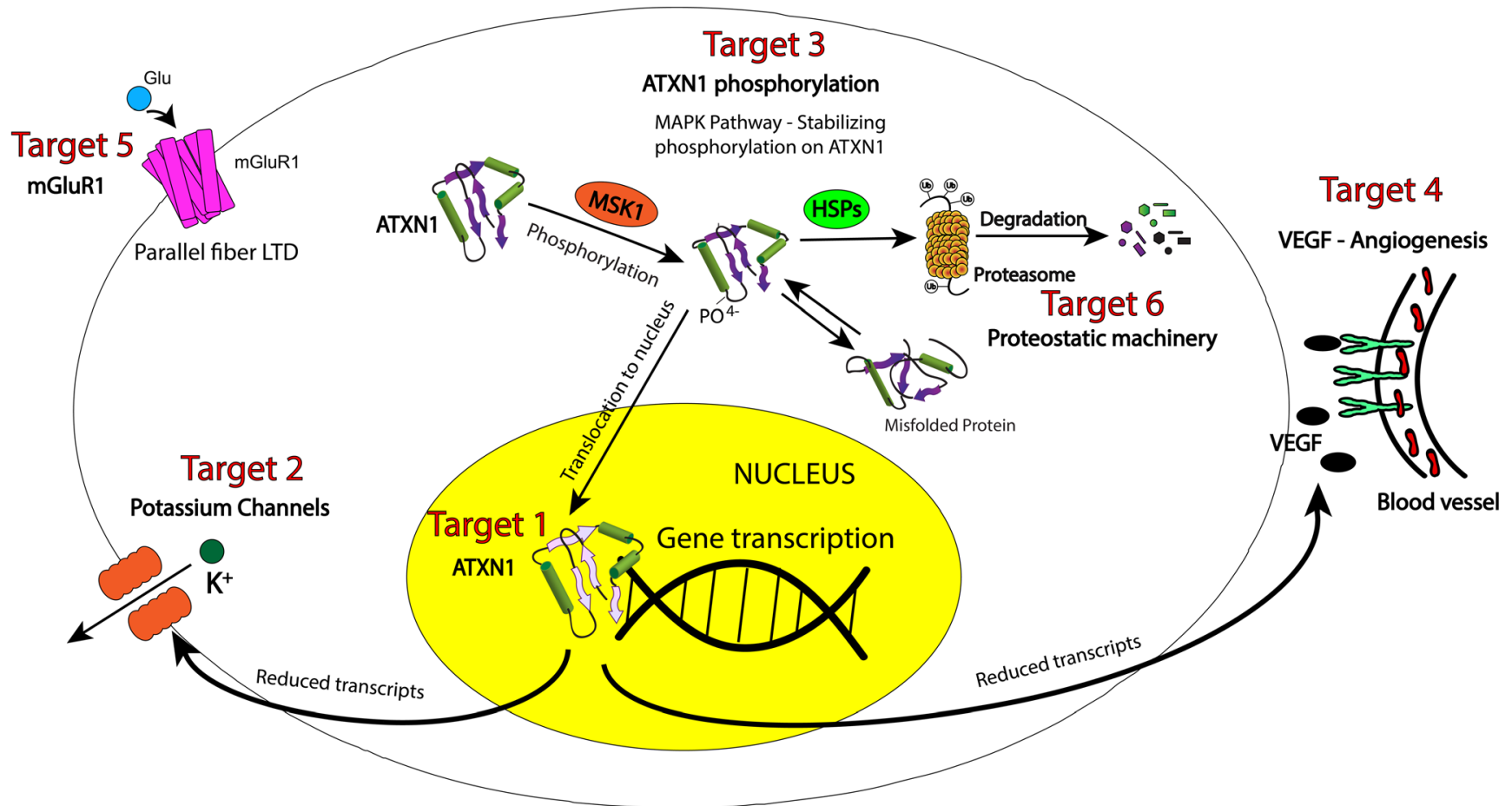

Fig. 1 Therapeutic targets for SCA1. Although polyQ expansion of ATXN1 underlies the pathophysiology of SCA1, there are many pathways that are disrupted, contributing to debilitating symptoms as well as disease progression. Several of these pathways present viable targets for disease modification and symptom improvement. ATXN1 translocates to the nucleus to influence gene transcription. Transcriptional targets that are particularly relevant to disease include

improving motor recovery in mouse models of disease [25]. It is important also, therefore, to examine targets, with respect to disease stage.

\section{Target 1: Mutant ATXN1}

Given that SCA1 is a monogenic disease, the obvious strategy is to target ATXN1 itself. What is unclear, however, is whether reducing ATXN1 levels is beneficial at all stages of disease. In a conditional transgenic mouse model of SCA1, halting mutant ATXN1 expression early in disease results in reversal of motor dysfunction. Halting mutant ATXN1 expression later in disease, resulted in prevention of progression, without reversal of motor dysfunction, even though no cell loss was present at this stage of disease [25]. Similarly, gene suppression strategies are effective in improving motor dysfunction in a knockin model of SCA1, but only when the intervention is at the onset of motor dysfunction [21]. A recent study has identified transcriptional changes detectable as early as a week after birth in SCA1-knockin mice. These transcriptional changes in cerebellar stem cells result in alterations in neuronal circuitry of the developing cerebellum [26]. It is possible that these structural changes that occur early in development will preclude complete reversibility of motor dysfunction even in the potassium channels and vascular endothelial growth factor A (VEGF). Mutant ATXN1 can be targeted directly, or through targeting the kinases in the RAS-MAPK-MSK1 pathway, which enhance the stability of ATXN1 through its phosphorylation. Other targets relevant to disease biology are the metabotropic glutamate receptor (mGluR1) and proteostasis

absence of cell loss. Also, we do not know the long-term consequences of removing wild-type ATXN1 from its physiologic tasks and targeting strictly polyQ ATXN1 still leaves only one functioning ATXN1 allele, without clear knowledge of haplosufficiency. It is therefore likely important to target functional output pathways to pursue symptom control in parallel with disease-modifying therapy.

\section{Antisense Oligonucleotides}

Short, single-stranded nucleic acids, antisense oligonucleotides (ASOs) disrupt RNA in a variety of means, including altering RNA binding protein interactions, splicing, translation, and prompting interaction with $\mathrm{RNaseH}$ and subsequent cleavage [27]. ASOs against superoxide dismutase (SOD1) have shown promise in ALS trials [28] and nusinersin (Spinraza), an ASO for spinal muscular atrophy (SMA1), was recently approved by the FDA [29]. Data from the knockin model of SCA1 with ASOs is promising [21], as is the case in mouse models of SCA2 [30] and SCA3 [31]. Early clinical trial data for Huntington Disease (HD) patients is also promising [32], suggesting that this is a viable avenue for targeting polyQ diseases. 


\section{CRISPR}

CRISPR/Cas9 systems are effective in genetically modifying single nucleotide polymorphisms (SNPs), creating gene deletions, or exon skipping through use of high fidelity homologydirected repair and nonhomologous end joining with specific guide RNAs [33, 34]. Despite their promise as a therapeutic strategy, these systems have hurdles with targeted delivery [35], particularly into the CNS (though newer adenovirus, lentivirus, and nanoparticle packaging strategies are promising), and no clear data for usage in SCAs exists. Further, recent studies demonstrating wide-spread genome rearrangements from CRISPR/Cas9 treatment raises serious concerns about off-target effects [36, 37].

\section{RNA Interference: Stereotactic Delivery of si/shRNAs and miRNAs}

Use of miRNAs has also been suggested as means of translational regulation. The ATXN1 gene was found to have several miRNA binding sites in the 3 'UTR $[38,39]$. This was validated in a SCA1 cell model [40] but use of these miRNAs led to increased cytotoxicity, potentially related to reduction of wildtype ATXN1 function.

shRNAs and siRNAs targeting mutant ATXN1 have been successfully transduced into cerebellar Purkinje cells using an adeno-associated vector (AAV) model [41]. In SCA1 mice, this led to reduced ATXN1 inclusions, restored cerebellar morphology, and improved motor function. Single injections of AAV si/shRNAs into the DCN were able to reduce ATXN1 mRNAs [42], improve SCA1 phenotype, and did not result in long-term side effects, thereby supportive of potential clinical use.

\section{Limitations}

Although genetic strategies may attempt to target the root cause of disease, there are several limitations to this approach, including typically a delay in diagnosis, and therefore timing of the treatment to when symptoms are already overt. There is likely some element of pathology that has already taken place and developmental changes in cerebellar circuitry would already be present. Further, there are intrinsic functions of ATXN1 that may be impaired if wild-type ATXN1 is targeted in addition to mutant ATXN1. For example, Atxn $1^{-/}$mice show impaired water maze and rotarod performance [43] and similar cerebellar changes as SCA1 models [44]. Atxn1[154Q] knockin mice on an Atxn1 knockout background have a worse phenotype compared to Atxn1 [154Q] on an Atxn1 wild-type background [45]. Allele specific polyQ ATXN1 reduction using the si/shRNA approach, without impairing wild-type ATXN1 function, is promising, although delivery to the CNS in humans is a hurdle that remains to be surmounted.

\section{Target 2: Potassium Channels}

Although polyQ ATXN1 is the driving force behind SCA1, there are many downstream pathways that are disrupted, resulting in cerebellar Purkinje neuron and brainstem nuclei dysfunction. A particularly relevant change that has been intensively studied is ion channel dysfunction in cerebellar Purkinje neurons [46, 47]. In several other SCAs, Purkinje neurons exhibit altered spiking regularity or rate $[17,18,48$, 49], a phenotype shown to arise from potassium channel dysfunction and loss of the after-hyperpolarization potential [50, 51]. In mouse models of SCA1, restoration of ion channel function improves motor dysfunction [19, 47, 52]. Recovery of ion channel dysfunction by overexpressing potassium channels also leads to delayed Purkinje neuron degeneration, particularly when treatment is started early in disease [19, 47]. Large conductance calcium-activated (BK), small conductance calcium-activated (SK), and inwardly rectifying (Kir) potassium channels have been identified as potential therapeutic targets in SCA1.

\section{4-Aminopyridine}

4-Aminopyridine (4-AP) was approved in 2010 for the improvement of gait instability and motor fatigue in multiple sclerosis $[53,54]$. This compound was subsequently shown to normalize cerebellar Purkinje cell firing in SCA1 [46] and SCA6 mouse models [55], and also to acutely improve motor dysfunction. Unfortunately, this compound blocks a wide array of potassium channels at higher doses leading to proconvulsant effects [53]. Also, 4-AP blocks Kv3 potassium channels potently [56], and there would thus be concern about long-term safety with this agent to treat symptoms in ataxia as loss-of-function mutations in Kv3.3 result in a degenerative cerebellar ataxia [57]. While it does indicate that potassium channels may serve as a target in disease, safety concerns with 4-AP will limit its long-term use in patients with any SCA.

\section{Combination of Chlorzoxazone and Baclofen}

Strong evidence exists for necessity of targeting more than one potassium channel in SCA1. A combination of chlorzoxazone and baclofen to target calcium-activated potassium and Kir channels may in fact be required. Chlorzoxazone is a known activator of both $\mathrm{BK}$ and $\mathrm{SK}$ channels $[58,59]$ while baclofen, a $\mathrm{GABA}_{\mathrm{B}}$ agonist, potentiates a sub-threshold activated potassium current in Purkinje neurons [60]. Neither compound alone was able to restore repetitive spiking in Purkinje cells in a transgenic mouse model of SCA1 but the combination was effective 
[52]. Use of another SK channel agonist, SKA-31 [61], in combination with baclofen provided similar results [52], lending credence to the importance of targeting several potassium channels. Importantly, the combination of baclofen and chlorzoxazone improved motor dysfunction in the transgenic SCA1 model, and also appeared to be tolerated in patients with ataxia [52]. Baclofen alone was unable to improve motor dysfunction in this model of SCA1 [19]. Chlorzoxazone is of low potency, however, and is thus vulnerable to off-target effects. Novel compounds with improved specificity and activity on calciumactivated potassium channels are therefore needed.

\section{Riluzole}

Best known for its use in amyotrophic lateral sclerosis (ALS) $[62,63]$, riluzole is a multifunctional benzothiazole that may have a role in SCA therapy. Although it was initially thought to work solely on glutamate signaling, it is now clear that riluzole blocks sodium channels, inhibits NMDA receptors, and also activates potassium channels [64-66]. The latter finding, and specifically its ability to activate SK channels suggested potential utility in ameliorating ataxia symptoms. In a heterogeneous group of patients with ataxia, riluzole treatment led to modest improvements of clinically validated ataxia rating scales $[67,68]$. Currently, a multicenter phase III trial of a pro-drug of riluzole (NCT03701399) and a single site phase III trial of riluzole (NCT03347334) are underway respectively in subjects with polyQ SCAs and SCA2.

\section{Target 3: Kinases Phosphorylating ATXN1}

Parallel cell-based and Drosophila genetic screens have been used to identify pathways that result in reduction of ATXN1 levels [69]. These data have shown that components of the RAS-MAPK-MSK pathway facilitate a stabilizing phosphorylation at the S776 residue of both wild-type and polyQ ATXN1 [70]. Inhibition of this pathway, either through siRNA knockdown of MEK, MSK1, or PAK1 leads to decreased polyQ ATXN1 levels, improved motor performance, and partial recovery of cerebellar Purkinje cell morphology in a SCA1 knockin mouse model $[69,71]$. Inhibiting multiple nodes of the pathway was shown to be additive in ATXN1 reduction. While a promising avenue for treatment, this approach again focuses on reducing ATXN1 levels, and would seem to affect both wild-type and polyQ forms. Additionally, targeting components of the MAPK pathway may lead to significant off-target effects given its ubiquity in cellular signaling throughout the body.

\section{Lithium}

Lithium has long been known to have effects on various neurological diseases and is discussed in relation to difficult-totreat neurodegenerative disorders, with a postulated mechanism of action through inhibition of glycogen synthase kinase $3[72,73]$. In a mouse model of SCA1, lithium resulted in significant improvement of motor coordination, learning, and memory [74]. Further, it led to reduced neuronal degeneration. These findings led to a phase I trial in SCA1 patients (NCT00683943, results not published) and SCA2 [75] and a phase II study in SCA3 $[75,76]$. Following the safety study in SCA1 in 2008, lithium has not been advanced to therapy trials for SCA1. Lithium was safe in SCA3, but did not slow disease progression [76].

\section{Target 4: VEGF}

Using transcriptional profiling in cerebellar Purkinje neurons, vascular endothelial growth factor A (VEGF) was identified to be downregulated in SCA1 knockin mice [77]. ATXN1 was shown to dose-dependently downregulate VEGF levels, which led to reduced cerebellar function through reduced vascular proliferation. VEGF overexpression was also shown to ameliorate the SCA1 phenotype, specifically leading to reduced cerebellar degeneration and improved performance on the rotarod. Intrathecal administration of recombinant VEGF into SCA1 models also showed similar results. Synthetic VEGF peptide amphiphiles that can assemble into nanoparticles and activate the VEGF receptor improved regularity of Purkinje cell firing [78], in addition to improved motor function and pathological changes. These nanoparticles have been shown to permeate brain tissue and have a half-life of nearly 4 weeks. An intrathecal delivery route was, however, needed for these nanoparticles. VEGF has been suggested as a promising neuroprotective strategy for other neurodegenerative disorders such as ALS [79]. Systemically delivered growth factors have had disappointing results in early ALS clinical trials [80], however, likely in part due to dose-limited side effects. It remains to be seen as to whether a viable delivery strategy, at therapeutic concentrations of VEGF, is possible without side effects in humans.

\section{Target 5: mGluR1}

Metabotropic glutamate receptor (mGluR1) signaling has been shown to be reduced in ATXN1 mice, in parallel with Purkinje cell dysfunction [81, 82]. Loss of mGluR1 led to reduced short- and long-term plasticity in cerebellar Purkinje cell firing resulting in poor motor learning. This led to the idea that enhancing mGluR1 could lead to recovery of cerebellar function, shown by injection of Ro0711401, a positive allosteric modulator that improved motor symptoms in SCA1 
knockin mice [83]. However, mGluR1's function may be more complex as other studies have shown that levels and activity were increased in SCA1 transgenic mice. A negative allosteric modulator, JNJ16259685 systemically delivered to ATXN1 transgenic mice improved motor dysfunction [81]. Thus, mGluR's involvement in SCA1 is clearly diseasestage dependent and thus its viability as a target is uncertain.

\section{Target 6: Proteostatic Machinery}

It is well established that neurons suffer from a decline in function of proteostatic machinery with aging [84] and that this decline can predispose one to neurodegenerative diseases [85]. Misfolded polyQ proteins provide an added stress to the cellular environment that is often neurotoxic. Thus, improving the proteostatic clearance of misfolded or aggregated proteins may be an avenue for treatment of neurodegenerative diseases.

An important component of neuronal proteostasis is the ubiquitin-proteasome system, which has also been implicated in other polyQ diseases. There is significant evidence to suggest that polyQ proteins are not properly ubiquitinated as they can form amyloid fibrils and are improperly removed by the proteasome [86]. Extensive work has been done on components of this system, including inhibiting deubiquitinases, augmenting ubiquitin ligases, and modifying chaperone activity $[87,88]$, with the last category perhaps having the most evidence. Indeed, several studies have shown that small molecules working to enhance molecular chaperone function can decrease polyQ forming proteins through diverting these aggregates via various proteostatic mechanisms [89-91]. Despite the potential benefits in polyQ SCAs, the biological rationale for compounds targeting this pathway in SCA1 is weak, suggesting that aberrant proteostasis may be less important as a therapeutic strategy.

\section{Other Therapeutic Strategies}

\section{Stem Cell-Based Therapy}

Implantation of embryonic cerebellar cells into SCA1 transgenic mice has been shown to improve motor impairment [92]. This benefit only lasted approximately 3 months however, after which cerebellar degeneration resumed. A subsequent study using neural precursor cells (NPCs) showed improved behavior and increased Purkinje cell survival [93], but only in later stage disease when uptake was enhanced, and it was not clear that these NPCs adopted cerebellar Purkinje cell morphology. Mesenchymal stem cells have also been used and have been shown to suppress PC dendritic atrophy and improved motor coordination in mouse models of SCA1 [94].
Dosing, delivery, and identifying a consistent population of stem cells in humans limit clinical studies with these agents.

\section{Deep Brain Stimulation}

The advent of electrical stimulation on subcortical targets has dramatically altered the course of both essential tremor and iPD. Deep brain stimulation (DBS) has proven efficacy in improving tremor, rigidity, and bradykinesia and minimizing motor fluctuations and dyskinesias in iPD [95]. These results raise the possibility to use DBS to modify cerebellar pathways in SCA. A preliminary study using DBS in the deep cerebellar nuclei (DCN) suggests improved motor function in a mouse model of SCA1 [96]. In Brazil, a clinical trial is underway examining the results of DBS versus sham surgery on improving chronic cerebellar ataxia (NCT03341416). Of note, this trial does not require a specific SCA diagnosis and involves a small number of subjects (10 subjects). The results should, however, prove both interesting and as a potential proof of concept.

\section{Tracking Treatment Outcomes}

Measuring treatment effect is an area sorely in need of development. The Scale for the Assessment and Rating of Ataxia (SARA) is widely used [97], easily performed in under $10 \mathrm{~min}$, and compared to other clinical scales has the most validation in SCA patients [98, 99]. However, there is some subjectivity in the scoring scheme, potential variability between examiners, and patients may perform better at an office appointment than at home, a "white-coat" manifestation of the Hawthorne Effect. To this end, many have suggested use of wearable technology to track gait instability and movements to obtain more reliable longitudinal data rather than isolated data points $[100,101]$. This technology is relatively new and still developing, and at times can be cumbersome for patients.

\section{Biomarkers-Tracking Target Engagement and Treatment Efficacy}

In addition to clinical examination of SCA patients, objective biomarkers have been long sought-after to provide unbiased longitudinal data as well as another method by which to track treatment response. An obvious marker might be detection of SCA polyQ proteins in CSF, and this has been shown to be feasible for another polyQ protein, huntingtin [102]. CSF levels of tau, $\alpha$-synuclein, DJ-1, and glial fibrillary acidic protein (GFAP) have been measured in patients with SCA and multiple system atrophy [103]. Tau levels were increased in patients with SCA2 and MSA, while $\alpha$-synuclein was decreased in SCA1 patients with higher SARA scores. None of these trends, however, reached statistical significance. 
Additional preclinical studies to identify methods to reliably detect ATXN1 with high sensitivity are still pending. Serological labs are also being pursued but are still far from clinical utility.

Imaging biomarkers provide another avenue of not only monitoring disease progression, but also examining patterns of involvement specific to SCA1. Several studies have shown metabolic changes and dopaminergic dysfunction in SCAs via positron emission tomography (PET) imaging. Given concerns over PET availability and radiation exposure, magnetic resonance (MR)-based platforms have been pursued [104]. MR spectroscopy (MRS) has identified changes in mutation carriers of SCA1 prior to onset of motor symptoms $[105,106]$. MRS is a noninvasive method for studying regional metabolic differences in SCA patients, tracking longitudinal decline and potential response to therapy. Another MR-based modality is voxel-based morphometry. Atrophy progression using this MR modality is more sensitive than clinical decline [107].

\section{Clinical Trials: Understanding Pitfalls and Moving Forward}

While preclinical studies have enhanced understanding of disease and identified novel therapy targets, the means of bringing a drug from bench to bedside is complex, especially in orphan diseases such as SCA1. Clinical trials depend on several relevant factors, including patient population, sample size, and effect size. The lower the impact of the treatment, the larger the sample needs to be to reach statistical significance. Previous studies have elegantly described the relationship between these two variables and have suggested that appropriate trials for SCA can be done with patient sizes from 70 to 300 $[4,108]$. As a trial size of even 70 patients is typically beyond the scope of most single institutions, it is important to use the resources of networks such as the European Integrated Project on Spinocerebellar Ataxias (EUROSCA) and the Clinical
Research Consortium for Spinocerebellar Ataxias (CRCSCA).

Applying the constraints of traditional clinical trials limits our ability to design trials in rare and difficult-to-treat diseases. It is therefore important to examine the design of trials for SCA1 (Fig. 2). Firstly, what patients should be included? It is easiest to run trials specific to SCA1 (SCA1 vs placebo). Genetic strategies directed towards ATXN1 gene suppression must clearly be directed specifically to this population. Other therapeutic strategies directed towards shared downstream dysfunction would allow grouping SCAs, and also identify medications that may be more broadly applicable than merely SCA1. Secondly, what effect size should we be powering studies to detect? It is important to have more information regarding the relationship between specific components of SARA and physician and patient reported functional measures. Powering studies to detect specified changes in SARA alone may not yield therapies that are clinically meaningful. The use of wearable technologies and more objective measures of function are needed in parallel with SARA [100, 101]. While this technology is still in infantile stages, it should certainly be incorporated into clinical trial structure as it becomes more readily available. Further development of serum and imaging biomarkers that are tied to functional measures would allow more objective data points, so that smaller sample sizes in trials may yet reach statistical and clinical significance.

\section{Conclusions}

Our goals in treating patients with SCA1 are quite clearimprove symptoms, delay progression, and reverse degenerative changes if possible. Recent developments have identified several promising targets. The complexities of polyQ diseases suggest, however, that the appropriate treatment strategy will involve a combinatorial approach, involving

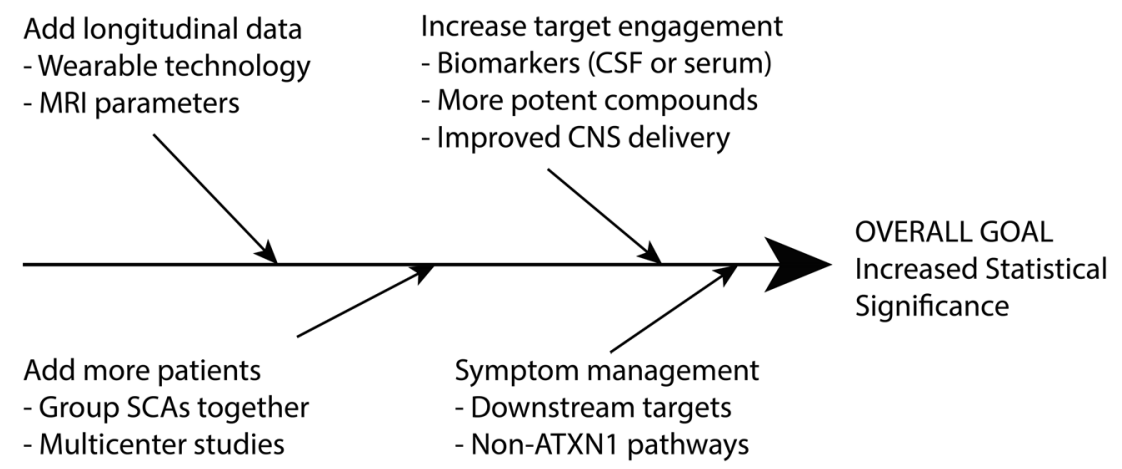

Fig. 2 Improving clinical trial structure in SCAs. An outline of methods that can be used to increase the statistical significance of clinical trials in rare diseases such as SCA1 is provided. Because achieving statistical significance in clinical trials for SCAs is difficult, we must rethink the design of traditional trials. For example, when the treatment target is shared with other SCAs, SCA1 may be grouped with other SCAs. By creative reorganization and addressing symptoms as well as underlying disease processes, we can more easily bring compounds from bench to bedside 
genetic and nongenetic strategies, and also including treatment focused on improving symptoms. Development of novel biomarkers tied to functional measures is necessary to refine clinical trial design, so that robust trials can be conducted with small sample sizes.

Acknowledgments Financial support for the authors' research was provided by the US National Institute of Neurological Disorders and Stroke (R01 NS085054), and the US National Ataxia Foundation.

Required Author Forms Disclosure forms provided by the authors are available with the online version of this article.

\section{References}

1. Shakkottai VG, Fogel BL. Clinical neurogenetics: autosomal dominant spinocerebellar ataxia. Neurol Clin, 31(4), 987-1007 (2013).

2. Durr A. Autosomal dominant cerebellar ataxias: polyglutamine expansions and beyond. Lancet Neurol, 9(9), 885-894 (2010).

3. Ashizawa T, Figueroa KP, Perlman SL et al. Clinical characteristics of patients with spinocerebellar ataxias 1,2,3 and 6 in the US; a prospective observational study. Orphanet J Rare Dis, 8, 177 (2013).

4. Jacobi H, du Montcel ST, Bauer P et al. Long-term disease progression in spinocerebellar ataxia types 1,2,3, and 6: a longitudinal cohort study. Lancet Neurol, 14(11), 1101-1108 (2015).

5. Robitaille Y, Schut L, Kish SJ. Structural and immunocytochemical features of olivopontocerebellar atrophy caused by the spinocerebellar ataxia type 1 (SCA-1) mutation define a unique phenotype. Acta Neuropathol, 90(6), 572-581 (1995).

6. Seidel K, Siswanto S, Brunt ER, den Dunnen W, Korf HW, Rub U. Brain pathology of spinocerebellar ataxias. Acta Neuropathol, 124(1), 1-21 (2012).

7. Ma J, Wu C, Lei J, Zhang X. Cognitive impairments in patients with spinocerebellar ataxia types 1,2 and 3 are positively correlated to the clinical severity of ataxia symptoms. Int J Clin Exp Med, 7(12), 5765-5771 (2014).

8. Sokolovsky N, Cook A, Hunt H, Giunti P, Cipolotti L. A preliminary characterisation of cognition and social cognition in spinocerebellar ataxia types 2, 1, and 7. Behav Neurol, 23(1-2), 17-29 (2010).

9. Menon RP, Nethisinghe $\mathrm{S}$, Faggiano $\mathrm{S}$ et al. The role of interruptions in polyQ in the pathology of SCA1. PLoS Genet, 9(7), e1003648 (2013).

10. Yue S, Serra HG, Zoghbi HY, Orr HT. The spinocerebellar ataxia type 1 protein, ataxin-1, has RNA-binding activity that is inversely affected by the length of its polyglutamine tract. Hum Mol Genet, 10(1), 25-30 (2001).

11. Lam YC, Bowman AB, Jafar-Nejad P et al. ATAXIN-1 interacts with the repressor Capicua in its native complex to cause SCA1 neuropathology. Cell, 127(7), 1335-1347 (2006).

12. Tsuda H, Jafar-Nejad H, Patel AJ et al. The AXH domain of Ataxin-1 mediates neurodegeneration through its interaction with Gfi-1/Senseless proteins. Cell, 122(4), 633-644 (2005).

13. Klement IA, Skinner PJ, Kaytor MD et al. Ataxin-1 nuclear localization and aggregation: role in polyglutamine-induced disease in SCA1 transgenic mice. Cell, 95(1), 41-53 (1998).

14. Irwin S, Vandelft M, Pinchev D et al. RNA association and nucleocytoplasmic shuttling by ataxin-1. J Cell Sci, 118(Pt 1), 233-242 (2005).
15. Duvick L, Barnes J, Ebner B et al. SCA1-like disease in mice expressing wild-type ataxin-1 with a serine to aspartic acid replacement at residue 776. Neuron, 67(6), 929-935 (2010).

16. Emamian ES, Kaytor MD, Duvick LA et al. Serine 776 of ataxin-1 is critical for polyglutamine-induced disease in SCA1 transgenic mice. Neuron, 38(3), 375-387 (2003).

17. Hansen ST, Meera P, Otis TS, Pulst SM. Changes in Purkinje cell firing and gene expression precede behavioral pathology in a mouse model of SCA2. Hum Mol Genet, 22(2), 271-283 (2013).

18. Shakkottai VG, do Carmo Costa M, Dell'Orco JM, Sankaranarayanan A, Wulff H, Paulson HL. Early changes in cerebellar physiology accompany motor dysfunction in the polyglutamine disease spinocerebellar ataxia type 3. J Neurosci, 31(36), 13002-13014 (2011).

19. Chopra R, Bushart DD, Shakkottai VG. Dendritic potassium channel dysfunction may contribute to dendrite degeneration in spinocerebellar ataxia type 1. PLoS One, 13(5), e0198040 (2018).

20. Globas C, du Montcel ST, Baliko L et al. Early symptoms in spinocerebellar ataxia type 1, 2, 3, and 6. Mov Disord, 23(15), 2232-2238 (2008).

21. Friedrich J, Kordasiewicz HB, O'Callaghan B et al. Antisense oligonucleotide-mediated ataxin-1 reduction prolongs survival in SCA1 mice and reveals disease-associated transcriptome profiles. JCI Insight, 3(21). pii: 123193. https://doi.org/10.1172/jci.insight. 123193 (2018).

22. Driessen TM, Lee PJ, Lim J. Molecular pathway analysis towards understanding tissue vulnerability in spinocerebellar ataxia type 1 . eLife, 7. pii:e39981. https://doi.org/10.7554/eLife.39981 (2018).

23. Fahn S, Oakes D, Shoulson I et al. Levodopa and the progression of Parkinson's disease. N Engl J Med, 351(24), 2498-2508 (2004).

24. Barchet TM, Amiji MM. Challenges and opportunities in CNS delivery of therapeutics for neurodegenerative diseases. Expert Opin Drug Deliv, 6(3), 211-225 (2009).

25. Zu T, Duvick LA, Kaytor MD et al. Recovery from polyglutamine-induced neurodegeneration in conditional SCA1 transgenic mice. J Neurosci, 24(40), 8853-8861 (2004).

26. Edamakanti CR, Do J, Didonna A, Martina M, Opal P. Mutant ataxin 1 disrupts cerebellar development in spinocerebellar ataxia type 1. J Clin Invest, 128(6), 2252-2265 (2018).

27. van Ommen GJ, Aartsma-Rus A. Advances in therapeutic RNAtargeting. New Biotechnol, 30(3), 299-301 (2013).

28. Miller TM, Pestronk A, David W et al. An antisense oligonucleotide against SOD1 delivered intrathecally for patients with SOD1 familial amyotrophic lateral sclerosis: a phase 1, randomised, firstin-man study. Lancet Neurol, 12(5), 435-442 (2013).

29. Finkel RS, Mercuri E, Darras BT et al. Nusinersen versus Sham Control in Infantile-Onset Spinal Muscular Atrophy. N Engl J Med, 377(18), 1723-1732 (2017).

30. Scoles DR, Meera P, Schneider MD et al. Antisense oligonucleotide therapy for spinocerebellar ataxia type 2. Nature, 544(7650), 362-366 (2017)

31. McLoughlin HS, Moore LR, Chopra R et al. Oligonucleotide therapy mitigates disease in spinocerebellar ataxia type 3 mice. Ann Neurol, 84(1), 64-77 (2018).

32. Tabrizi SJ, Leavitt BR, Landwehrmeyer GB et al. Targeting Huntingtin Expression in Patients with Huntington's Disease. N Engl J Med, 380(24), 2307-2316 (2019).

33. Stella S, Alcon P, Montoya G. Class 2 CRISPR-Cas RNA-guided endonucleases: Swiss Army knives of genome editing. Nat Struct Mol Biol, 24(11), 882-892 (2017).

34. Murugan K, Babu K, Sundaresan R, Rajan R, Sashital DG. The Revolution Continues: Newly Discovered Systems Expand the CRISPR-Cas Toolkit. Mol Cell, 68(1), 15-25 (2017).

35. Lino CA, Harper JC, Carney JP, Timlin JA. Delivering CRISPR: a review of the challenges and approaches. Drug Deliv, 25(1), 1234-1257 (2018). 
36. Pawluk A, Davidson AR, Maxwell KL. Anti-CRISPR: discovery, mechanism and function. Nat Rev Microbiol, 16(1), 12-17 (2018).

37. Maxwell KL. The Anti-CRISPR Story: A Battle for Survival. Mol Cell, 68(1), 8-14 (2017).

38. Lee Y, Samaco RC, Gatchel JR, Thaller C, Orr HT, Zoghbi HY. miR-19, miR-101 and miR-130 co-regulate ATXN1 levels to potentially modulate SCA1 pathogenesis. Nat Neurosci, 11(10), 1137-1139 (2008).

39. Persengiev S, Kondova I, Otting N, Koeppen AH, Bontrop RE. Genome-wide analysis of miRNA expression reveals a potential role for miR-144 in brain aging and spinocerebellar ataxia pathogenesis. Neurobiol Aging, 32(12), 2316 e2317-2327 (2011).

40. Keiser MS, Monteys AM, Corbau R, Gonzalez-Alegre P, Davidson BL. RNAi prevents and reverses phenotypes induced by mutant human ataxin-1. Ann Neurol, 80(5), 754-765 (2016).

41. Xia H, Mao Q, Eliason SL et al. RNAi suppresses polyglutamineinduced neurodegeneration in a model of spinocerebellar ataxia. Nat Med, 10(8), 816-820 (2004).

42. Keiser MS, Boudreau RL, Davidson BL. Broad therapeutic benefit after RNAi expression vector delivery to deep cerebellar nuclei: implications for spinocerebellar ataxia type 1 therapy. Mol Ther, 22(3), 588-595 (2014).

43. Matilla A, Roberson ED, Banfi S et al. Mice lacking ataxin-1 display learning deficits and decreased hippocampal paired-pulse facilitation. J Neurosci, 18(14), 5508-5516 (1998).

44. Crespo-Barreto J, Fryer JD, Shaw CA, Orr HT, Zoghbi HY. Partial loss of ataxin-1 function contributes to transcriptional dysregulation in spinocerebellar ataxia type 1 pathogenesis. PLoS Genet, 6(7), e1001021 (2010).

45. Lim J, Crespo-Barreto J, Jafar-Nejad P et al. Opposing effects of polyglutamine expansion on native protein complexes contribute to SCA1. Nature, 452(7188), 713-718 (2008).

46. Hourez R, Servais L, Orduz D et al. Aminopyridines correct early dysfunction and delay neurodegeneration in a mouse model of spinocerebellar ataxia type 1. J Neurosci, 31(33), 11795-11807 (2011).

47. Dell'Orco JM, Wasserman AH, Chopra R et al. Neuronal Atrophy Early in Degenerative Ataxia Is a Compensatory Mechanism to Regulate Membrane Excitability. J Neurosci, 35(32), 11292 11307 (2015).

48. Kasumu AW, Hougaard C, Rode F et al. Selective positive modulator of calcium-activated potassium channels exerts beneficial effects in a mouse model of spinocerebellar ataxia type 2 . Chem Biol, 19(10), 1340-1353 (2012).

49. Dell'Orco JM, Pulst SM, Shakkottai VG. Potassium channel dysfunction underlies Purkinje neuron spiking abnormalities in spinocerebellar ataxia type 2. Hum Mol Genet, 26(20), 39353945 (2017).

50. Sausbier M, Hu H, Arntz $\mathrm{C}$ et al. Cerebellar ataxia and Purkinje cell dysfunction caused by $\mathrm{Ca} 2+-$ activated $\mathrm{K}+$ channel deficiency. Proc Natl Acad Sci U S A, 101(25), 9474-9478 (2004).

51. Edgerton JR, Reinhart PH. Distinct contributions of small and large conductance $\mathrm{Ca} 2+-$ activated $\mathrm{K}+$ channels to rat Purkinje neuron function. J Physiol, 548(Pt 1), 53-69 (2003).

52. Bushart DD, Chopra R, Singh V, Murphy GG, Wulff H, Shakkottai VG. Targeting potassium channels to treat cerebellar ataxia. Ann Clin Transl Neurol, 5(3), 297-314 (2018).

53. Bever CT, Jr., Young D, Anderson PA et al. The effects of 4aminopyridine in multiple sclerosis patients: results of a randomized, placebo-controlled, double-blind, concentration-controlled, crossover trial. Neurology, 44(6), 1054-1059 (1994).

54. Goodman AD, Brown TR, Krupp LB et al. Sustained-release oral fampridine in multiple sclerosis: a randomised, double-blind, controlled trial. Lancet, 373(9665), 732-738 (2009).
55. Jayabal S, Chang HH, Cullen KE, Watt AJ. 4-aminopyridine reverses ataxia and cerebellar firing deficiency in a mouse model of spinocerebellar ataxia type 6. Sci Rep, 6, 29489 (2016).

56. Grissmer S, Nguyen AN, Aiyar J et al. Pharmacological characterization of five cloned voltage-gated $\mathrm{K}+$ channels, types Kv1.1, $1.2,1.3,1.5$, and 3.1 , stably expressed in mammalian cell lines. Mol Pharmacol, 45(6), 1227-1234 (1994).

57. Waters MF, Minassian NA, Stevanin G et al. Mutations in voltagegated potassium channel $\mathrm{KCNC} 3$ cause degenerative and developmental central nervous system phenotypes. Nat Genet, 38(4), 447-451 (2006)

58. Liu YC, Lo YK, Wu SN. Stimulatory effects of chlorzoxazone, a centrally acting muscle relaxant, on large conductance calciumactivated potassium channels in pituitary GH3 cells. Brain Res, 959(1), 86-97 (2003).

59. Cao Y, Dreixler JC, Roizen JD, Roberts MT, Houamed KM. Modulation of recombinant small-conductance $\mathrm{Ca}(2+)$-activated $\mathrm{K}(+)$ channels by the muscle relaxant chlorzoxazone and structurally related compounds. J Pharmacol Exp Ther, 296(3), 683-689 (2001).

60. Tabata T, Haruki S, Nakayama H, Kano M. GABAergic activation of an inwardly rectifying $\mathrm{K}+$ current in mouse cerebellar Purkinje cells. J Physiol, 563(Pt 2), 443-457 (2005).

61. Sankaranarayanan A, Raman G, Busch C et al. Naphtho[1,2d]thiazol-2-ylamine (SKA-31), a new activator of $\mathrm{KCa} 2$ and $\mathrm{KCa} 3.1$ potassium channels, potentiates the endothelium-derived hyperpolarizing factor response and lowers blood pressure. Mol Pharmacol, 75(2), 281-295 (2009).

62. Bensimon G, Lacomblez L, Meininger V. A controlled trial of riluzole in amyotrophic lateral sclerosis. ALS/Riluzole Study Group. N Engl J Med, 330(9), 585-591 (1994).

63. Lacomblez L, Bensimon G, Leigh PN, Guillet P, Meininger V. Dose-ranging study of riluzole in amyotrophic lateral sclerosis. Amyotrophic Lateral Sclerosis/Riluzole Study Group II. Lancet, 347(9013), 1425-1431 (1996).

64. Kretschmer BD, Kratzer U, Schmidt WJ. Riluzole, a glutamate release inhibitor, and motor behavior. Naunyn Schmiedeberg's Arch Pharmacol, 358(2), 181-190 (1998).

65. Beltran-Parrazal L, Charles A. Riluzole inhibits spontaneous $\mathrm{Ca} 2+$ signaling in neuroendocrine cells by activation of $\mathrm{K}+$ channels and inhibition of $\mathrm{Na}+$ channels. Br J Pharmacol, 140(5), 881888 (2003).

66. Cao YJ, Dreixler JC, Couey JJ, Houamed KM. Modulation of recombinant and native neuronal SK channels by the neuroprotective drug riluzole. Eur J Pharmacol, 449(1-2), 47-54 (2002).

67. Ristori G, Romano S, Visconti A et al. Riluzole in cerebellar ataxia: a randomized, double-blind, placebo-controlled pilot trial. Neurology, 74(10), 839-845 (2010).

68. Romano S, Coarelli G, Marcotulli C et al. Riluzole in patients with hereditary cerebellar ataxia: a randomised, double-blind, placebocontrolled trial. Lancet Neurol, 14(10), 985-991 (2015).

69. Park J, Al-Ramahi I, Tan Q et al. RAS-MAPK-MSK1 pathway modulates ataxin 1 protein levels and toxicity in SCA1. Nature, 498(7454), 325-331 (2013).

70. Zoghbi HY, Orr HT. Pathogenic mechanisms of a polyglutaminemediated neurodegenerative disease, spinocerebellar ataxia type 1 . J Biol Chem, 284(12), 7425-7429 (2009).

71. Bondar VV, Adamski CJ, Onur TS et al. PAK1 regulates ATXN1 levels providing an opportunity to modify its toxicity in spinocerebellar ataxia type 1. Hum Mol Genet, 27(16), 28632873 (2018)

72. Lazzara CA, Kim YH. Potential application of lithium in Parkinson's and other neurodegenerative diseases. Front Neurosci, 9, 403 (2015).

73. Forlenza OV, De-Paula VJ, Diniz BS. Neuroprotective effects of lithium: implications for the treatment of Alzheimer's disease and 
related neurodegenerative disorders. ACS Chem Neurosci, 5(6), 443-450 (2014).

74. Watase K, Gatchel JR, Sun Y et al. Lithium therapy improves neurological function and hippocampal dendritic arborization in a spinocerebellar ataxia type 1 mouse model. PLoS Med, 4(5), e182 (2007).

75. Sacca F, Puorro G, Brunetti A et al. A randomized controlled pilot trial of lithium in spinocerebellar ataxia type 2. J Neurol, 262(1), 149-153 (2015).

76. Saute JA, de Castilhos RM, Monte TL et al. A randomized, phase 2 clinical trial of lithium carbonate in Machado-Joseph disease. Mov Disord, 29(4), 568-573 (2014).

77. Cvetanovic M, Patel JM, Marti HH, Kini AR, Opal P. Vascular endothelial growth factor ameliorates the ataxic phenotype in a mouse model of spinocerebellar ataxia type 1. Nat Med, 17(11), 1445-1447 (2011).

78. $\mathrm{Hu}$ YS, Do J, Edamakanti CR et al. Self-assembling vascular endothelial growth factor nanoparticles improve function in spinocerebellar ataxia type 1. Brain, 142(2), 312-321 (2019).

79. Storkebaum E, Lambrechts D, Dewerchin M et al. Treatment of motoneuron degeneration by intracerebroventricular delivery of VEGF in a rat model of ALS. Nat Neurosci 8(1), 85-92 (2005).

80. Sorenson EJ, Windbank AJ, Mandrekar JN et al. Subcutaneous IGF-1 is not beneficial in 2-year ALS trial. Neurology, 71(22), 1770-1775 (2008)

81. Power EM, Morales A, Empson RM. Prolonged Type 1 Metabotropic Glutamate Receptor Dependent Synaptic Signaling Contributes to Spino-Cerebellar Ataxia Type 1. J Neurosci, 36(18), 4910-4916 (2016).

82. Shuvaev AN, Hosoi N, Sato Y, Yanagihara D, Hirai H. Progressive impairment of cerebellar mGluR signalling and its therapeutic potential for cerebellar ataxia in spinocerebellar ataxia type 1 model mice. J Physiol, 595(1), 141-164 (2017).

83. Notartomaso S, Zappulla C, Biagioni F et al. Pharmacological enhancement of mGlul metabotropic glutamate receptors causes a prolonged symptomatic benefit in a mouse model of spinocerebellar ataxia type 1. Mol Brain, 6, 48 (2013).

84. Brehme $\mathrm{M}$, Voisine $\mathrm{C}$, Rolland $\mathrm{T}$ et al. A chaperome subnetwork safeguards proteostasis in aging and neurodegenerative disease. Cell Rep, 9(3), 1135-1150 (2014).

85. Hartl FU, Bracher A, Hayer-Hartl M. Molecular chaperones in protein folding and proteostasis. Nature, 475(7356), 324-332 (2011).

86. Sakahira H, Breuer P, Hayer-Hartl MK, Hartl FU. Molecular chaperones as modulators of polyglutamine protein aggregation and toxicity. Proc Natl Acad Sci U S A, 99 Suppl 4, 16412-16418 (2002).

87. Nagai Y, Fujikake N, Popiel HA, Wada K. Induction of molecular chaperones as a therapeutic strategy for the polyglutamine diseases. Curr Pharm Biotechnol, 11(2), 188-197 (2010).

88. Nath SR, Lieberman AP. The Ubiquitination, Disaggregation and Proteasomal Degradation Machineries in Polyglutamine Disease. Front Mol Neurosci, 10, 78 (2017).

89. Reis SD, Pinho BR, Oliveira JMA. Modulation of Molecular Chaperones in Huntington's Disease and Other Polyglutamine Disorders. Mol Neurobiol, 54(8), 5829-5854 (2017).

90. Ding Y, Adachi H, Katsuno M et al. BIIB021, a synthetic Hsp90 inhibitor, induces mutant ataxin-1 degradation through the activation of heat shock factor 1. Neuroscience, 327, 20-31 (2016).

91. Williams AJ, Knutson TM, Colomer Gould VF, Paulson HL. In vivo suppression of polyglutamine neurotoxicity by $\mathrm{C}$ terminus of Hsp70-interacting protein (CHIP) supports an aggregation model of pathogenesis. Neurobiol Dis, 33(3), 342-353 (2009).
92. Kaemmerer WF, Low WC. Cerebellar allografts survive and transiently alleviate ataxia in a transgenic model of spinocerebellar ataxia type-1. Exp Neurol, 158(2), 301-311 (1999).

93. Chintawar S, Hourez R, Ravella A et al. Grafting neural precursor cells promotes functional recovery in an SCA1 mouse model. J Neurosci, 29(42), 13126-13135 (2009).

94. Matsuura S, Shuvaev AN, Iizuka A, Nakamura K, Hirai H. Mesenchymal stem cells ameliorate cerebellar pathology in a mouse model of spinocerebellar ataxia type 1. Cerebellum, 13(3), 323-330 (2014).

95. Wagle Shukla A, Okun MS. Surgical treatment of Parkinson's disease: patients, targets, devices, and approaches. Neurotherapeutics, 11(1), 47-59 (2014).

96. Vedam-Mai V, McFarland K, Nathu R et al. Effectiveness of Deep Brain Stimulation (DBS) in Mice with Spinocerebellar Ataxia (SCA1). [abstract]. Mov Disord, 32 (suppl 2). https://www. mdsabstracts.org/abstract/effectiveness-of-deep-brainstimulation-dbs-in-mice-with-spinocerebellar-ataxiasca1/. Accessed 15 July 2019 (2017).

97. Schmitz-Hubsch T, du Montcel ST, Baliko L et al. Scale for the assessment and rating of ataxia: development of a new clinical scale. Neurology, 66(11), 1717-1720 (2006)

98. Saute JA, Donis KC, Serrano-Munuera C et al. Ataxia rating scales-psychometric profiles, natural history and their application in clinical trials. Cerebellum, 11(2), 488-504 (2012).

99. Monte TL, Reckziegel ER, Augustin MC et al. NESSCA Validation and Responsiveness of Several Rating Scales in Spinocerebellar Ataxia Type 2. Cerebellum, 16(4), 852-858 (2017).

100. Hickey A, Gunn E, Alcock L et al. Validity of a wearable accelerometer to quantify gait in spinocerebellar ataxia type 6 . Physiol Meas, 37(11), N105-N117 (2016).

101. Subramony SH, Kedar S, Murray E et al. Objective home-based gait assessment in spinocerebellar ataxia. J Neurol Sci, 313(1-2), 95-98 (2012).

102. Southwell AL, Smith SE, Davis TR et al. Ultrasensitive measurement of huntingtin protein in cerebrospinal fluid demonstrates increase with Huntington disease stage and decrease following brain huntingtin suppression. Sci Rep, 5, 12166 (2015).

103. Brouillette AM, Oz G, Gomez CM. Cerebrospinal Fluid Biomarkers in Spinocerebellar Ataxia: A Pilot Study. Dis Markers, 2015, 413098 (2015).

104. Currie S, Hadjivassiliou M, Craven IJ, Wilkinson ID, Griffiths PD, Hoggard N. Magnetic resonance imaging biomarkers in patients with progressive ataxia: current status and future direction. Cerebellum, 12(2), 245-266 (2013).

105. Oz G, Kittelson E, Demirgoz D et al. Assessing recovery from neurodegeneration in spinocerebellar ataxia 1: Comparison of in vivo magnetic resonance spectroscopy with motor testing, gene expression and histology. Neurobiol Dis, 74, 158-166 (2015).

106. Oz G, Nelson CD, Koski DM et al. Noninvasive detection of presymptomatic and progressive neurodegeneration in a mouse model of spinocerebellar ataxia type 1. J Neurosci, 30(10), 3831-3838 (2010).

107. Reetz K, Costa AS, Mirzazade S et al. Genotype-specific patterns of atrophy progression are more sensitive than clinical decline in SCA1, SCA3 and SCA6. Brain, 136(Pt 3), 905-917 (2013).

108. Jacobi H, Reetz K, du Montcel ST et al. Biological and clinical characteristics of individuals at risk for spinocerebellar ataxia types 1, 2, 3, and 6 in the longitudinal RISCA study: analysis of baseline data. Lancet Neurol, 12(7), 650-658 (2013).

Publisher's Note Springer Nature remains neutral with regard to jurisdictional claims in published maps and institutional affiliations. 